\title{
Comparison of the present and planned operation of the SIS18 and the AGS Booster with intermediate charge state heavy ions
}

A. Smolyakov (ITEP), W. Fischer (BNL), C. Omet (GSI), P. Spiller (GSI)

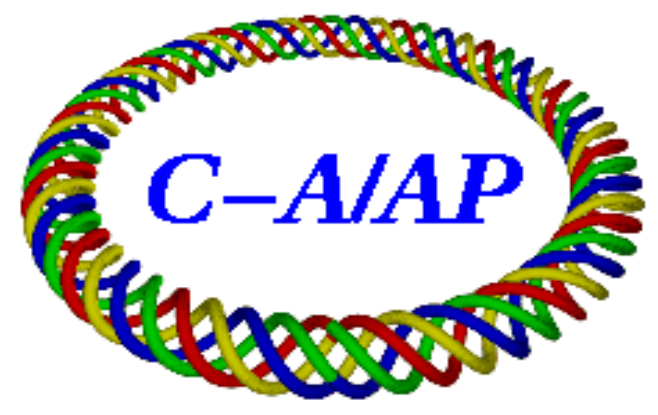

Collider-Accelerator Department Brookhaven National Laboratory

Upton, NY 11973 
Comparison of the present and planned operation of the SIS18 and the AGS Booster with intermediate charge state heavy ions

A. Smolyakov (ITEP); W. Fischer (BNL), C. Omet, P. Spiller (GSI)

$$
\text { ITEP, BNL, GSI }
$$

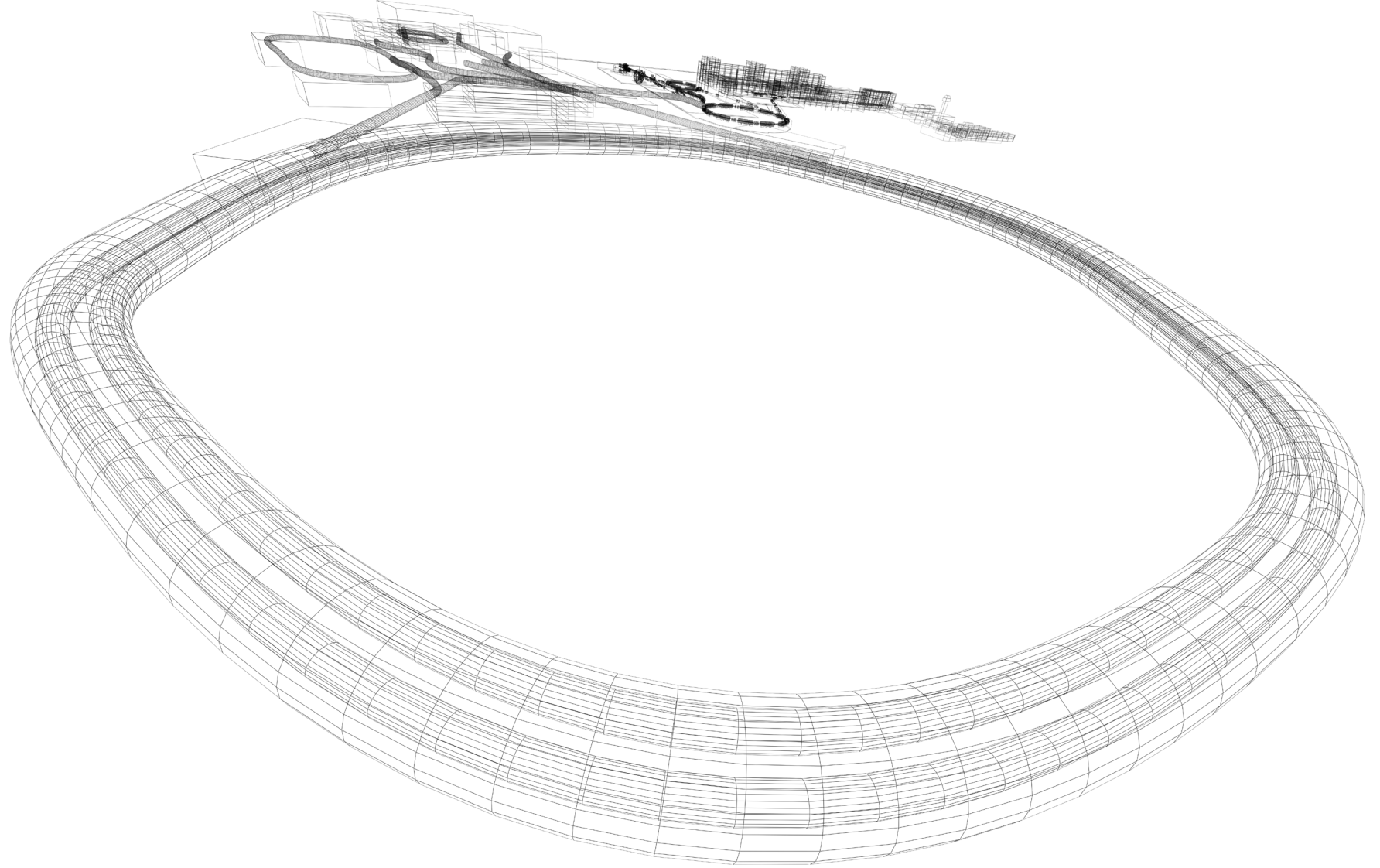

GSI-Acc-Report-2005-11-001

November 8, 2005 


\title{
Comparison of the present and planned operation of the SIS18 and the AGS Booster with intermediate charge state heavy ions
}

\author{
A. Smolyakov (ITEP), W. Fischer (BNL), C. Omet (GSI), P. Spiller (GSI)
}

November 7, 2005

\begin{abstract}
The heavy ion synchrotron SIS18 at GSI will be used in two different operation modes within the FAIR project [1]. One of these modes is the operation as a booster synchrotron for the planned SIS100. This mode will require high intensity operation with intermediate charge state heavy ion beams, in particular acceleration of $2.7 *$ $10^{11} U^{28+}$ ions per machine cycle.

However, the present UHV state does not allow an operation with intermediate charge state heavy ion beams with such intensities. Therefore an upgrade program has been defined involving several major technical systems. Apart from other goals, the upgrade of the UHV system shall stabilize the dynamic vacuum pressure and thereby enable the planned booster operation.

In the context of the planned operation of SIS18 and SIS100, the simulation code StrahlSim has been developed which couples the beam dynamics, the atomic physics processes and the induced vacuum phenomena. This code was used to carry out a comparison between the current and planned SIS18 operation and the operation of the AGS Booster at BNL.
\end{abstract}

\section{Comparison of the operation parameters of SIS18 and AGS Booster}

In the following tables a comparison of major SIS18 and AGS Booster operation parameters is given, divided into three groups: inherent synchrotron parameters (tab. 1), beam parameters (tab. 2) and vacuum parameters (tab. 3). The SIS18 data were taken from [2], [3] and [4], the AGS Booster data from [5] and [6].

Note that the present ramping rate of SIS18 is $1.3 \mathrm{~T} / \mathrm{s}$. It is planned to increase the ramp rate after finishing the new power connection to $4 \mathrm{~T} / \mathrm{s}$ in 2006 and up to $10 \mathrm{~T} / \mathrm{s}$ in 2009. However, calculations were carried out under the assumption of fast ramping with $10 \mathrm{~T} / \mathrm{s}$.

Note that the given SIS18 acceptance is defined by the UHV system and does not account for injection and extraction devices. 
Table 1: Synchrotron parameters for SIS18 and AGS Booster

\begin{tabular}{l|l|r|r} 
Parameter & unit & SIS18 & AGS Boooster \\
\hline Horizontal acceptance $\epsilon_{a c c, h}$ & $\mathrm{~mm}$ mrad & 250 & 185 \\
Vertical acceptance $\epsilon_{a c c, v}$ & $\mathrm{~mm}$ mrad & 95 & 87 \\
\hline Horizontal average Beta-function $\beta_{h}$ & $\mathrm{~m}$ & 9.37 & 9.0 \\
Vertical average Beta-function $\beta_{v}$ & $\mathrm{~m}$ & 11.96 & 9.0 \\
\hline Synchrotron circumference $L$ & $\mathrm{~m}$ & 216.8 & 201.78 \\
Ramp rate & $\mathrm{T} / \mathrm{s}$ & 10 & 8.3 \\
Injection duration & $\mu \mathrm{s}$ & 160 & 500 \\
\hline Systematic injection losses & $\%$ & 20 & 15 \\
Systematic RF-capture losses & $\%$ & 10 & 15
\end{tabular}

Table 2: Beam parameters for SIS18 and AGS Booster

\begin{tabular}{l|l|r|r} 
Parameter & unit & SIS18 & AGS Boooster \\
\hline Beam ion species, nuclear charge $Z$ & & $\mathrm{U}, Z=92$ & $\mathrm{Au}, Z=79$ \\
Charge state of the ion beams $q$ & $\mathrm{e}$ & $28+$ & $32+$ \\
\hline Injection energy $E_{\text {inj }}$ & $\mathrm{MeV} / \mathrm{u}$ & 11.4 & 0.92 \\
Extraction energy $E_{\text {ext }}$ & $\mathrm{MeV} / \mathrm{u}$ & 196.6 & 100.00 \\
\hline Horizontal emittance $\epsilon_{h}$ & $\mathrm{~mm} \mathrm{mrad}$ & 200 & 87 \\
Vertical emittance $\epsilon_{v}$ & $\mathrm{~mm} \mathrm{mrad}$ & 50 & 87 \\
\hline Injected number of particles & & up to $3 * 10^{11}$ & $2.6 * 10^{9} \ldots 6.1 * 10^{9}$
\end{tabular}


Table 3: Vacuum parameters for SIS18 and AGS Booster

\begin{tabular}{|c|c|c|c|}
\hline Parameter & unit & SIS18 & AGS Booster \\
\hline Base residual gas pressure $p_{0}$ & mbar & $5.0 * 10^{-11}$ & $2.7 * 10^{-11}$ \\
\hline Beam tube volume $V$ & $m^{3}$ & 4.0 & 2.2 \\
\hline Effective pumping speed $S_{\text {eff }}$ & $1 / \mathrm{s}$ & 2000 & 9600 \\
\hline Vacuum composition & & $\begin{array}{r}H_{2} 81.87 \% \\
C H_{4} 11.86 \% \\
C O \quad 3.02 \% \\
A r 3.25 \%\end{array}$ & $\begin{array}{l}\mathrm{H}_{2} 95 \% \\
\mathrm{CO} 5 \%\end{array}$ \\
\hline $\begin{array}{l}\text { Electron capture cross section } \\
\text { for beam ions at injection energy } \sigma_{c}\end{array}$ & $\mathrm{~cm}^{2} /$ atom & $\begin{array}{rl}{ }_{1} H & 1.4 * 10^{-22} \\
{ }_{6} C & 2.6 * 10^{-19} \\
{ }_{8} O & 8.0 * 10^{-19} \\
{ }_{18} A r & 4.1 * 10^{-18}\end{array}$ & $\begin{array}{l}{ }_{1} H \quad 3.6 * 10^{-17} \\
{ }_{6} C 5.6 * 10^{-16} \\
{ }_{8} O 5.8 * 10^{-16}\end{array}$ \\
\hline $\begin{array}{l}\text { Electron loss cross section } \\
\text { for beam ions at injection energy } \sigma_{l}\end{array}$ & $\mathrm{~cm}^{2} /$ atom & $\begin{array}{r}{ }_{1} H 4.7 * 10^{-18} \\
{ }_{6} C 2.8 * 10^{-17} \\
{ }_{8} O 3.7 * 10^{-17} \\
{ }_{18} A r 8.0 * 10^{-17}\end{array}$ & $\begin{array}{ll}{ }_{1} H & 1.1 * 10^{-18} \\
{ }_{6} C & 5.5 * 10^{-18} \\
{ }_{8} O & 6.8 * 10^{-18}\end{array}$ \\
\hline $\begin{array}{l}\text { Electron loss cross section } \\
\text { for beam ions at extraction energy } \sigma_{l}\end{array}$ & $\mathrm{~cm}^{2} /$ atom & $\begin{array}{r}{ }_{1} H 1.5 * 10^{-19} \\
{ }_{6} C 4.3 * 10^{-18} \\
{ }_{8} O 5.8 * 10^{-18} \\
{ }_{18} A r 1.2 * 10^{-17}\end{array}$ & $\begin{array}{l}{ }_{1} H \quad 1.3 * 10^{-19} \\
{ }_{6} C \quad 3.8 * 10^{-18} \\
{ }_{8} O 5.0 * 10^{-18}\end{array}$ \\
\hline $\begin{array}{l}\text { Residual gas ionization cross section } \\
\text { at injection energy } \sigma_{r}\end{array}$ & $c m^{2} /$ ion & $\begin{array}{rr}\mathrm{H}_{2} & 3.2 * 10^{-15} \\
\mathrm{CH}_{4} & 1.5 * 10^{-14} \\
\mathrm{CO} & 1.2 * 10^{-14} \\
\mathrm{Ar} & 1.2 * 10^{-14}\end{array}$ & $\begin{array}{c}\mathrm{H}_{2} 1.5 * 10^{-14} \\
\mathrm{CO} 3.2 * 10^{-14}\end{array}$ \\
\hline $\begin{array}{l}\text { Residual gas ionization cross section } \\
\text { at extraction energy } \sigma_{r}\end{array}$ & $\mathrm{~cm}^{2} /$ ion & $\begin{array}{rr}\mathrm{H}_{2} & 3.3 * 10^{-16} \\
\mathrm{CH}_{4} & 1.7 * 10^{-15} \\
\mathrm{CO} & 1.4 * 10^{-15} \\
\mathrm{Ar} & 1.5 * 10^{-15}\end{array}$ & $\begin{array}{r}H_{2} 7.2 * 10^{-16} \\
C O 3.0 * 10^{-15}\end{array}$ \\
\hline $\begin{array}{l}\text { Desorption coefficient for beam ions } \\
\text { under grazing incidence } \eta\end{array}$ & & $2.8 * 10^{4}$ & $7.0 * 10^{3}$ \\
\hline $\begin{array}{l}\text { Desorption coefficient for residual gas } \\
\text { molecules under perpendicular incidence }\end{array}$ & $1 / \mathrm{mol}$ & 10 & 10 \\
\hline
\end{tabular}


The electron capture and electron loss cross-sections for Uranium ions were taken from [7], whereas the cross-sections for the Gold ions were calculated based on [8] and [9]. However, these parameters are not experimentally confirmed. Investigations have shown that the desorption coefficient scales with the electronic energy loss $(d E / d x)^{2}$. Different measurements have indicated that the desorption coefficient for low energetic $U^{28+}$ is in the range from 5000 to 30000 . Therefore we use the desorption coefficient as a fitting parameter in the range from $2160 \ldots 10000$ and apply a scaling according to the energy loss at the relevant beam energy (see tab. 4).

The planned upgrade of the SIS18 vacuum system shall lead to a static pressure of $p_{0} \approx$

Table 4: Electronic energy loss, calculated with SRIM [10]

\begin{tabular}{l|r|r|r}
$\mathrm{dE} / \mathrm{dx} / \mathrm{MeV} / \mathrm{mm}$ & AGS Booster & SIS18 & $\eta,(d E / d x)^{2}$-scaled \\
\hline at injection energy & $4.3 * 10^{4}$ & $7.2 * 10^{4}$ & 10000 \\
at extraction energy & $2.2 * 10^{4}$ & $2.0 * 10^{4}$ & 2160
\end{tabular}

$1 * 10^{-12}$ mbar [11]. It is planned to replace the dipole and quadrupole magnet chambers by new ones and develop a new bake-out system for higher bake-out temperature of $300^{\circ} \mathrm{C}$ (the existing one is operated up to $200^{\circ} \mathrm{C}$ ). In addition, NEG coating of all magnet chambers and the revision of all existing pumps shall provide the required high local and distributed pumping speed. Finally the upgraded effective pumping speed will be about $2 * 10^{5} \mathrm{l} / \mathrm{s}$, what is about 100 times higher than the total pumping speed at present.

Moreover, a collimation system [12], [13] is planned for beam losses generated by chargeexchange processes and prevent the desorbed ions and molecules from interacting with the circulating beam. Furthermore, a reduction of systematic beam losses, in particular injection losses to $5 \%$ and RF-capture losses to $1 \%$ is planned.

\section{Simulation results}

All the following calculations of the beam lifetime and pressure evolution were done using the code StrahlSim [13]. This code includes the simulation of different beam loss mechanisms like coulomb scattering, projectile and target ionization and couples these mechanisms to the induced gas desorption, the desorption collimation system and the resulting vacuum dynamics. Furthermore, realistic beam loss during injection, RF capture, acceleration and extraction in the AGS Booster and SIS18 acceleration cycle have been included. The lattice for the SIS18 was taken from [3] in WinAGILE format (suitable for StrahlSim) whereas the lattice for the AGS Booster was provided in MAD format [6] and then converted to WinAGILE.

In order to demonstrate the perspectives, simulations were carried out for higher beam intensities in the unchanged SIS18 and AGS Booster for three different initial beam intensities: $2.5 * 10^{9}$ ions, $3.0 * 10^{10}$ ions and $3.0 * 10^{11}$ ions. Furthermore, a comparison with the future SIS18 operation was carried out for an initial beam intensity of $3 * 10^{11}$, 
which is the goal value for the booster operation in the FAIR project.

\subsection{Present operation of SIS18 and AGS Booster}

In fig. 1, a comparison of StrahlSim results with measured beam intensity profiles in an AGS Booster cycle with an initial intensity of $2.6 * 10^{9}$ (which is achieved in standard operation) and $6.1 * 10^{9}$ ions is plotted. The calculated (ring averaged!) pressure profile is shown in fig 3 . In both cases, measured and simulated beam intensity profiles are in good agreement. No fast pressure measurement does exist for the $A u^{32+}$-case. However, in machine experiments with beam losses of about $3 * 10^{9} A u^{31+}$ ions, a maximum pressure in the order of $10^{-5} \mathrm{~Pa}$ was measured in the AGS Booster [14].

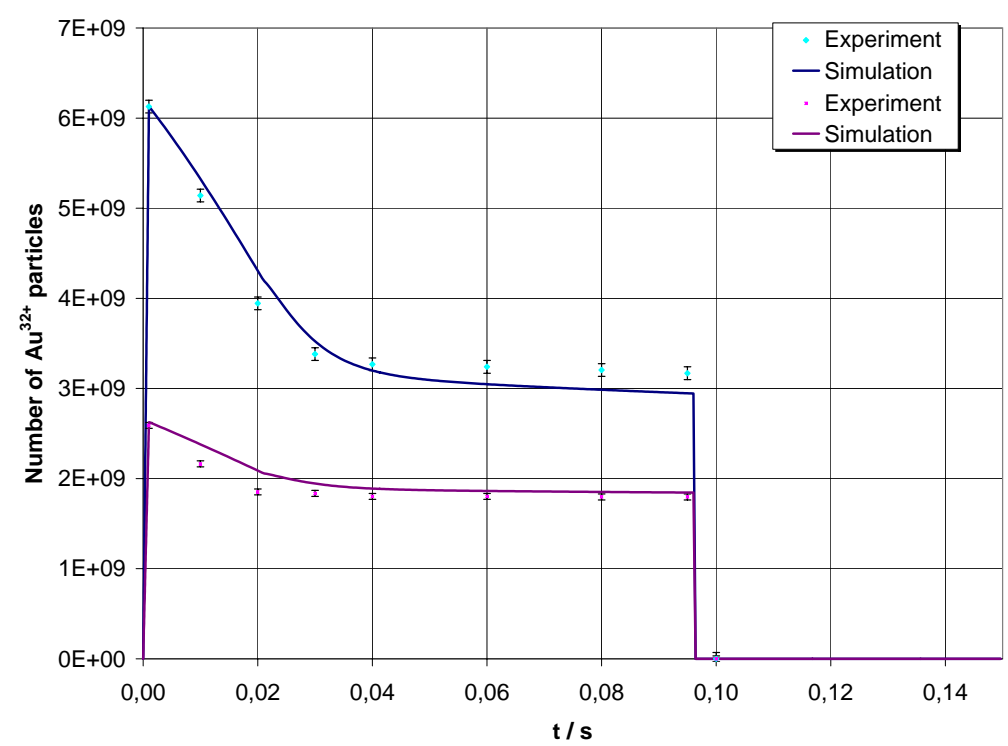

Figure 1: Calculated and measured beam intensity profiles in an AGS Booster cycle for different initial intensities 


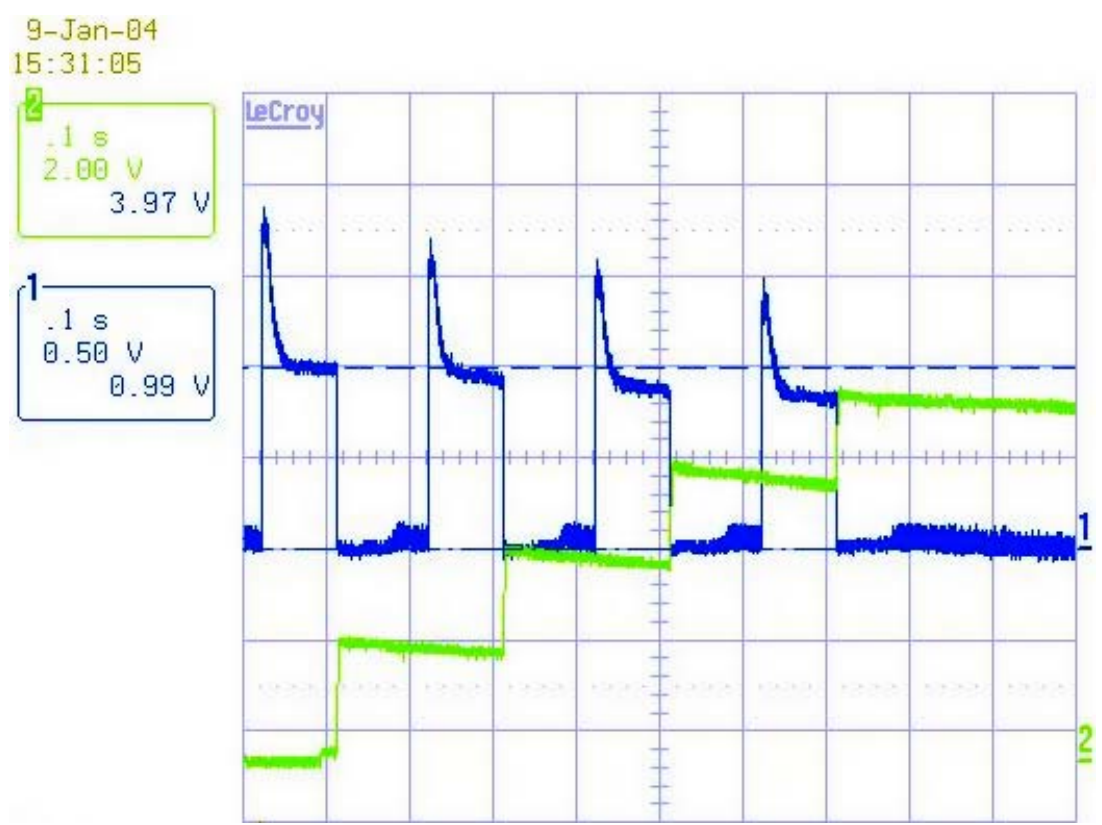

Figure 2: Measured beam intensity profiles in AGS Booster cycles (blue, compare fig. 1)

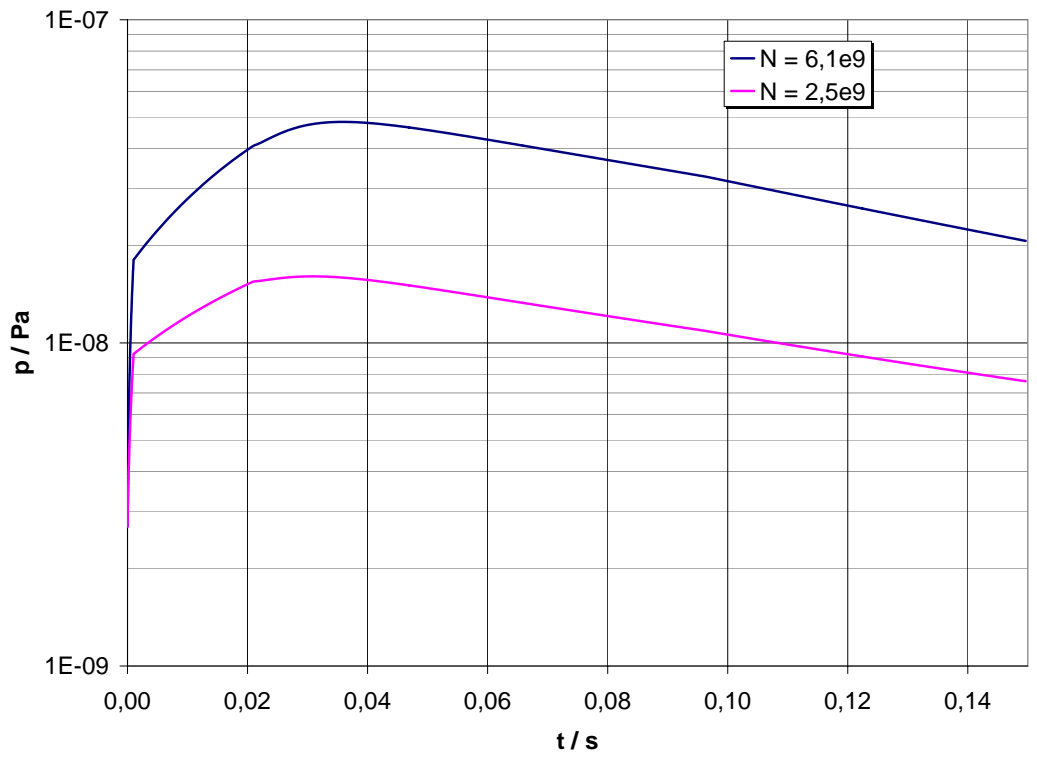

Figure 3: Calculated pressure profile in an AGS Booster cycle for different initial intensities 


\subsection{Current and planned operation of SIS18}

Fig. 4 and fig. 5 show a comparison of the present operation of SIS18 and AGS Booster with the planned operation of the SIS18 at beam intensities of $3 * 10^{10}$ and $3 * 10^{11}$ ions. For the planned SIS18 operation a lower base vacuum pressure of $p_{0}=1 * 10^{12}$ mbar is considered as well as higher effective pumping speed and lower systematic losses. The pressure evolution is plotted in fig. 6/7. Note the large pressure rise due to the injection losses.

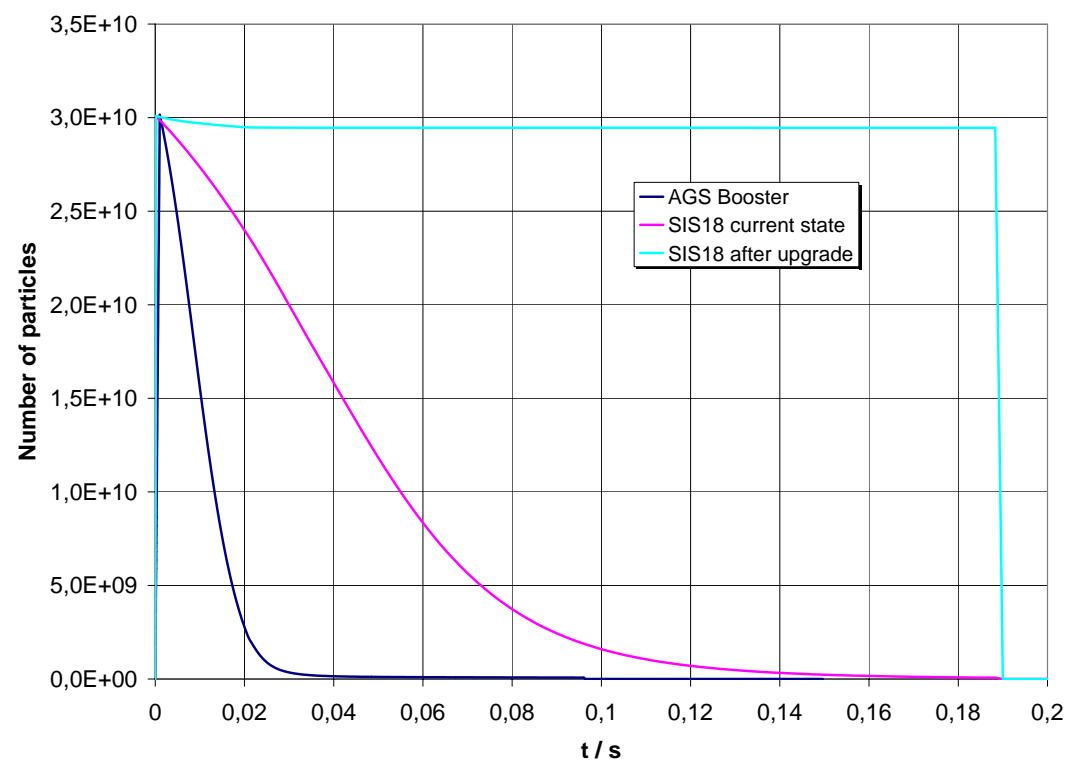

Figure 4: Predicted operation of SIS18 and AGS Booster at $3 * 10^{10}$ injected particles 


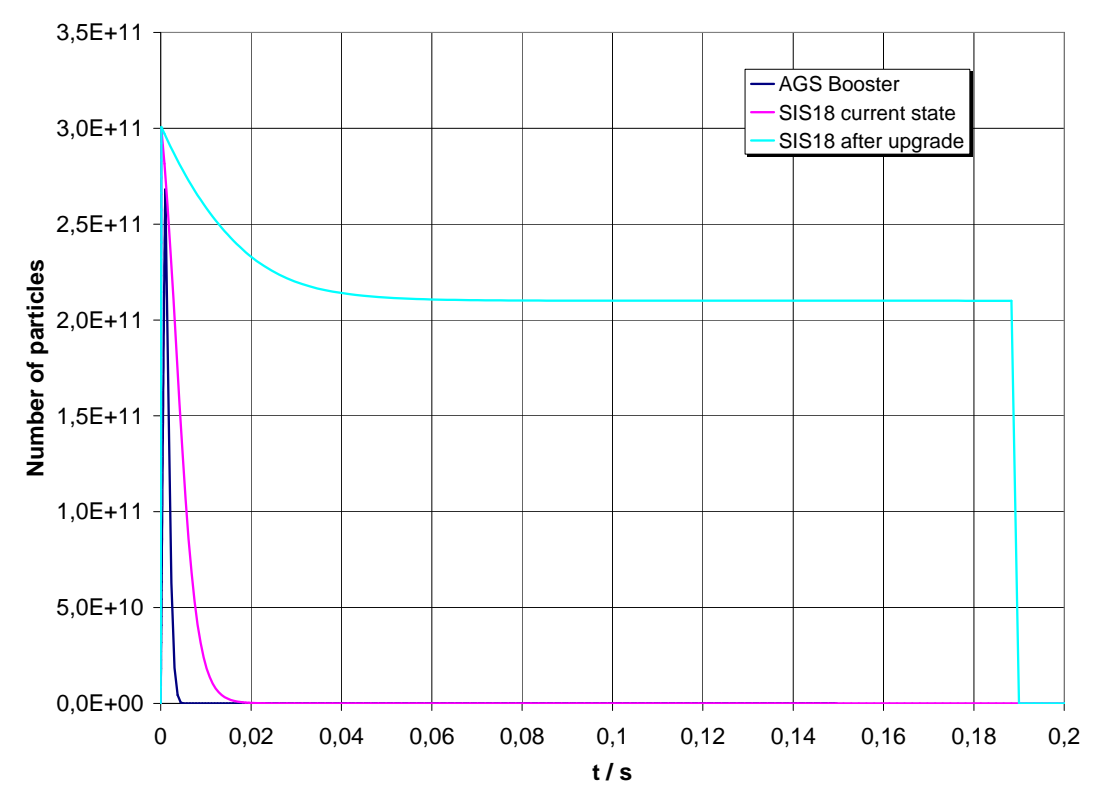

Figure 5: Predicted operation of SIS18 and AGS Booster at $3 * 10^{11}$ injected particles

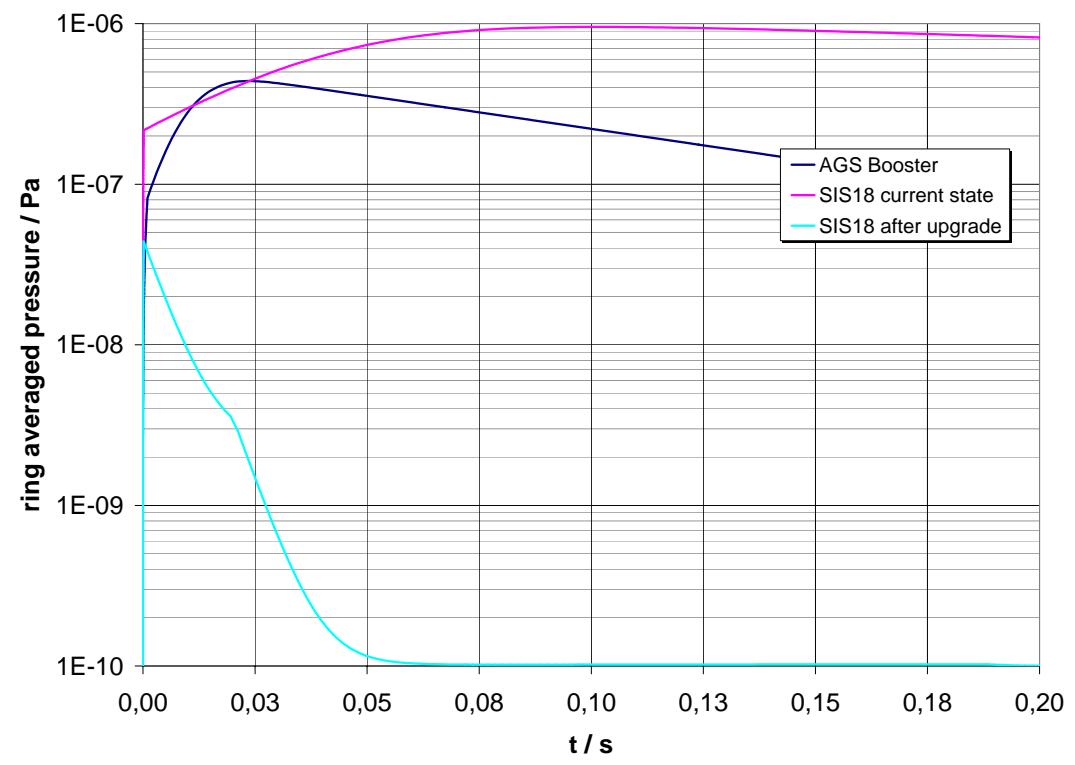

Figure 6: Predicted average pressure in SIS18 and AGS Booster at $3 * 10^{10}$ injected particles 


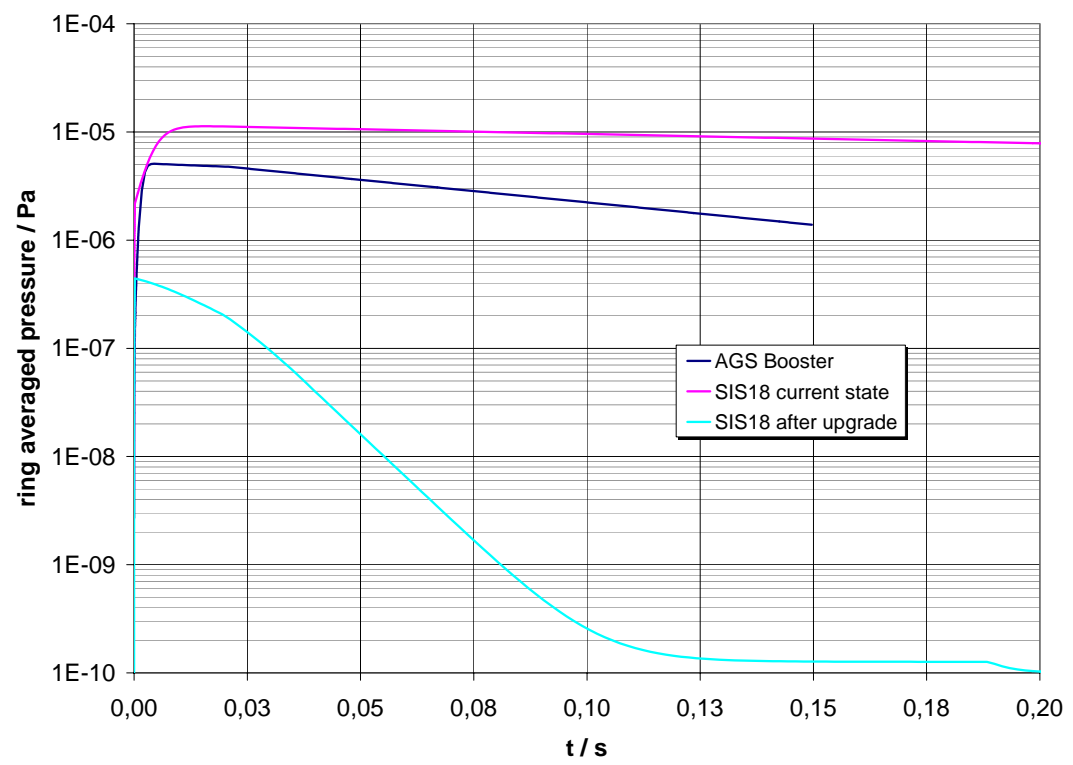

Figure 7: Predicted average pressure in SIS18 and AGS Booster at $3 * 10^{11}$ injected particles. Note the different scaling to fig. 6 . 


\section{Conclusions}

The comparison of the operation of SIS18 and AGS Booster with intermediate charge state heavy ions was carried out. The simulations carried out with the code StrahlSim show that the presently accelerated $A u^{32+}$ beam with an initial intensity of $6 * 10^{9} A u^{32+}$ ions is at the intensity limit at the AGS Booster. This result is fairly consistent with measurements of the beam intensity profile during the acceleration cycle. For SIS18 with its present UHV system the intensity limit for $U^{28+}$ beams appears to be 2 to 3 times higher, although it is in the same order of magnitude.

At higher initial intensities in both synchrotrons, the ion beam will be completely lost before the extraction if the UHV systems would remain unchanged. In this case the beam lifetime in the AGS Booster is evidently lower than in SIS18. This can be explained with much higher charge-exchange cross-sections at injection energies. In SIS18, the dominant process for $U^{28+}$ ions at the injection energy of $11.4 \mathrm{MeV} / \mathrm{u}$ is electron loss with cross sections of $10^{-18}$ to $10^{-17} \mathrm{~cm}^{2} /$ atom. In the AGS Booster the dominant process for $A u^{32+}$ ions at $0.92 \mathrm{MeV} / \mathrm{u}$ is electron capture with cross sections of $10^{-17}$ to $10^{-16} \mathrm{~cm}^{2} /$ atom. At similar base vacuum conditions and also similar and relatively high injection losses this leads to much higher beam losses in the AGS Booster.

Furthermore, the potential operation with higher intensities in the fully upgraded SIS18 and the present AGS Booster has been simulated. SIS18 after reaching the planned upgrade parameters shows quite encouraging results evidently allowing the acceleration of $U^{28+}$-ion beams with intensities of $10^{11}$. One should note that the injection losses (which occur during a $\mu s$ timescale) produce a very high pressure rise (the order of magnitude is nearly independent of the base pressure). 


\section{References}

[1] An international accelerator facility for beams of ions and antiprotons. http:// www.gsi.de/GSI-Future/cdr/, 2004. Conceptual Design Report.

[2] A. Smolyakov and P. Spiller. Beam Loss Calculations for U28+-ion beams interacting with the residual gas in SIS18 and SIS100. Note, GSI, 2004.

[3] C. Omet. Private communication.

[4] P. Spiller. Private communication.

[5] C.J. Gardner. Booster, AGS and RHIC parameters for the 2003-2004 RHIC Run. Note, BNL, 082003.

[6] W. Fischer. Private communication.

[7] R. E. Olsen. Projectile Electron Loss and Capture in $\mathrm{MeV} / \mathrm{u}$ Collisions of U28+ with H2, N2 and Ar. Phys. Lett., B, 2004.

[8] P. Spiller A. Smolyakov, C. Omet. Electron capture for fast ions in gas targets. Application of Schlachter scaling rule at nonrelativistic energies. Note, GSI, 09 2005.

[9] N. Madsen. Vacuum changes during accumulation of Pb54+ in LEIR. Note PS/DI 99-21, CERN, 111999.

[10] J.F. Ziegler. SRIM, Stopping and Range of Ions in Matter. http://www.srim.org, 2005.

[11] SIS18 Upgrade. Technical report, GSI, 2005.

[12] P. Spiller. GSI-SIS18-02-02. Internal note, GSI, 022002.

[13] C. Omet. Verlustrechnungen für Ionenstrahlen unter dynamischem Vakuum in Ringbeschleunigern. GSI Internal Report GSI-Acc-Report-2004-12-001, 2004.

[14] S.Y. Zhang and L.A. Ahrens. Gold beam losses at the AGS Booster injection. In Proceedings of the 1999 PAC, pages 3294-3296, 1999. 


\title{
A comparison of the AGS Booster operation with $\mathrm{Au}^{32+}$ ions at $0.92 \mathrm{MeV} / \mathrm{u}$ and $1.85 \mathrm{MeV} / \mathrm{u}$ injection energy.
}

\author{
A. Smolyakov, P. Spiller, C. Omet
}

The injection energy for $\mathrm{Au}^{32+}$ ions in AGS Booster synchrotron is $0.92 \mathrm{MeV} / \mathrm{u}$. At this energy the dominating process is electron capture [1]. In order to decrease the amount of electron-capture beam losses it was proposed to increase the injection energy and consequently decreasing the electron-capture cross-sections by a factor of 4-20 for the different species of residual gas atoms [2].

Using the StrahlSim simulation program [1], [3], a simulation was carried out in order to study the difference between the AGS Booster operation with $\mathrm{Au}^{32+}$ ion beams for both injection energies $(0.92 \mathrm{MeV} / \mathrm{u}$ and $1.85 \mathrm{MeV} / \mathrm{u})$ and initial numbers of ions $\left(3 \times 10^{9}\right.$ and $\left.6 \times 10^{9}\right)$. All other simulation parameters and conditions could be found in [1].

The simulation results are shown on the Fig. 1. It can be seen that with increased injection energy, we expect the number of beam particles at extraction growing by a factor of 2 .

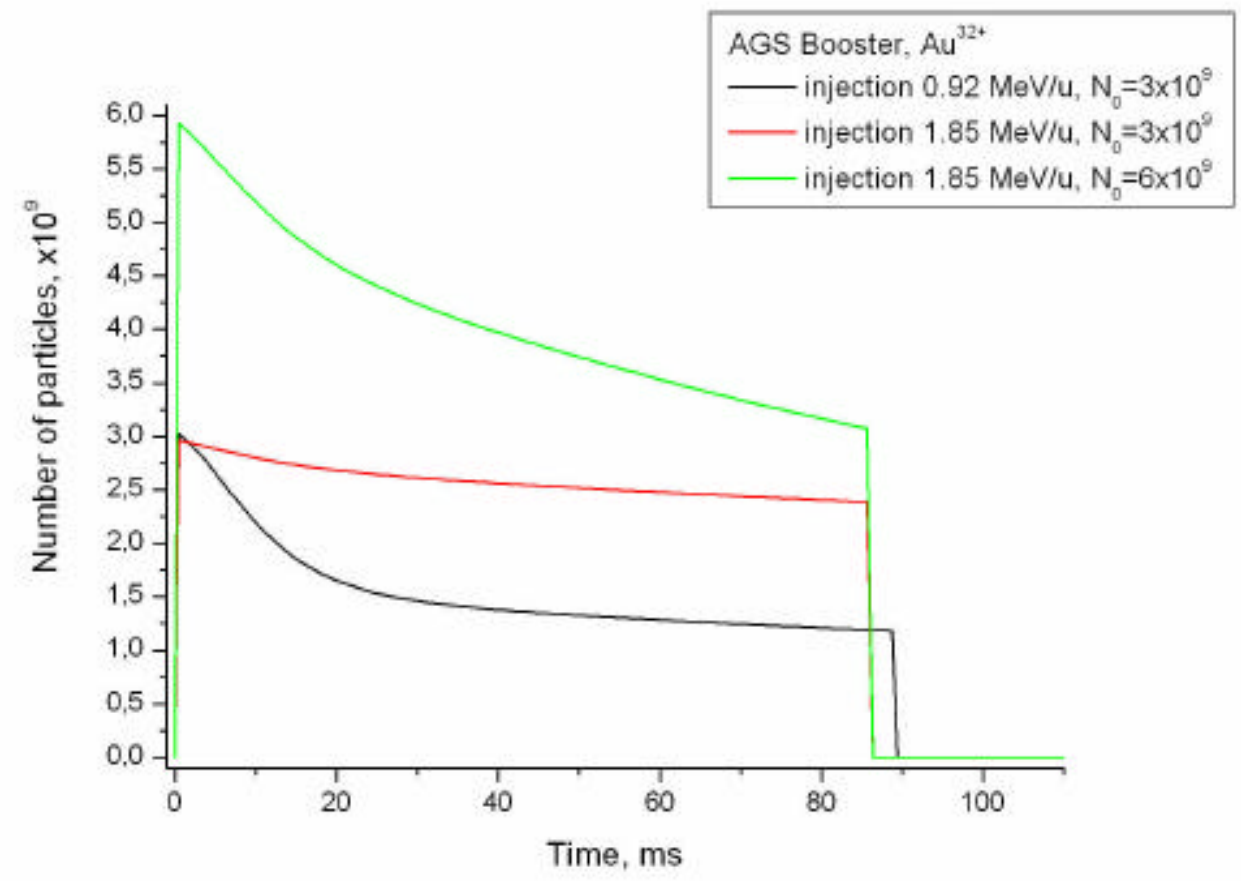

Fig. 1. A simulation of AGS Booster operation with $\mathrm{Au}^{32+}$ ion beam.

\section{References}

[1] Comparison of the present and planned operation of the SIS18 and the AGS Booster with intermediate charge state heavy ions, A. Smolyakov, W. Fischer, C. Omet, P. Spiller, GSI Internal Report GSI-Acc-Report-2005-11-01

[2] Electron capture for fast ions in gas targets. Application of Schlachter scaling rule at nonrelativistic energies. A. Smolyakov, C. Omet, P. Spiller, 09.2005

[3] Verlustrechnungen für Ionenstrahlen unter dynamischem Vakuum in Ringbeschleunigern, C. Omet, GSI Internal Report GSI-Acc-Report-2004-12-001 (2004) 